\title{
HPV infection in men
}

\author{
Joel M. Palefsky* \\ Department of Medicine, University of California, San Francisco, CA 94143, USA
}

\begin{abstract}
While much is known about the natural history of cervical human papillomavirus (HPV) infection and its consequences, including cervical intraepithelial neoplasia and cervical cancer, relatively little is known about the natural history of anogenital HPV infection and diseases in men. In part this reflects difficulties in penile sampling and visual assessment of penile lesions. Anal HPV infection and disease also remain poorly understood. Although HPV is transmitted sexually and infects the genitals of both sexes, the cervix remains biologically more vulnerable to malignant transformation than does the penis or anus in men. An understanding of male HPV infection is therefore important in terms of reducing transmission of HPV to women and improving women's health. However, it is also important due to the burden of disease in men, who may develop both penile and anal cancer, particularly among HIV-positive men who have sex with men. Improved sampling techniques of the male genitalia and cohort studies in progress should provide important information on the natural history of anogenital HPV infection and disease in men, including risk factors for HPV acquisition and transmission. The impact of HPV vaccination in women on male anogenital HPV infection will also need to be assessed.
\end{abstract}

Keywords: Penile HPV infection, anal HPV infection, penile intraepithelial neoplasia, anal intraepithelial neoplasia, penile cancer, anal cancer, condyloma, HIV

\section{Introduction}

The most common cause of HPV-associated mortality is cancer of the cervix, and most of the attention of clinicians and researchers has appropriately been directed toward this disease and its precursors. However, HPV infection of men is of great importance as well given that sexual transmission is the primary mode of spread to women. An understanding of anogenital HPV infection in men is therefore critical to reducing the risk of HPV transmission to women. Moreover, similar to women, men suffer the consequences of anogenital HPV infection in the form of anogenital condyloma, intraepithelial neoplasia and cancer. Recognition and treatment of anogenital HPV-associated lesions in men is critical to reducing the burden of disease in men and treatment of penile lesions has the potential, albeit as

*Address for correspondence: Joel Palefsky, M.D., 505 Parnassus Ave Room M1203, Box 0126, Department of Medicine, University of California, San Francisco, San Francisco, CA 94143, USA. Tel: + +1 415476 1574; Fax: +1 415476 0986; E-mail: joel.palefsky@ucsf.edu. yet unproven, to reduce the risk of HPV transmission to women.

The recent success of HPV vaccination to prevent initial HPV infection in women begs the question as to whether men should be vaccinated. Consistent with the above, the benefits could include reduced HPV transmission to women and increase in "herd immunity", as well as reduced penile and anal cancer incidence. Studies have not yet been published documenting efficacy of the vaccine in men, but these are in progress. Even in the absence of vaccinating men, however, a high penetrance of the vaccine in women would likely affect the prevalence and incidence of male anogenital disease in the future, and this will need to be the subject of further study.

\section{HPV infection and disease of the penis}

\subsection{Epidemiology of penile HPV infection}

Understanding the epidemiology of penile HPV infection and HPV-associated diseases has been challenging for a number of reasons. Unlike sampling a moist 
surface such as the cervix, reliable sampling of the dry, keratinized surface of the penis to obtain adequate numbers of cells has been difficult and poorly reproducible. Recently however, several investigators have been using better methods to collect cells, including the use of sandpaper or emery paper to loosen cells, resulting in better yields. This has improved our understanding of the prevalence and risk factors for penile HPV infection.

In one study of penile swabs obtained from 436 men attending a sexually transmitted disease clinic, the prevalence of HPV was $28.2 \%$. Oncogenic HPV types were found in $12.0 \%$ of participants, nononcogenic types were found in $14.8 \%$ of participants, multiple types were found in $6.1 \%$ of participants, and unknown types were found in $5.9 \%$ of participants [1]. In a study of male partners of women positive for HPV using the non-amplification-based Hybrid Capture ${ }^{\text {TM }}$ II (HCII) method, HC II analysis of penile brushings showed that $24 \%$ were positive in the glans, $44 \%$ in the prepuce internal surface, $30 \%$ in the distal urethra, $24 \%$ in the prepuce external surface, $12 \%$ in the scrotum and $8 \%$ in the anus [2].

In a study of 779 Mexican men requesting a vasectomy, exfoliated cells were obtained from the scrotum, the shaft of the penis and the top of the penis including the coronal sulcus, the glans and the opening of the meatus. Using PCR, the prevalence of any type of HPV was somewhat lower than in the $8.7 \%$ and the most commonly found HPV types were HPV59, 51, 6, 16 and 58. HPV positivity was highest among men below age $25(13.6 \%)$, and lowest among men aged 40 years or older $(6.0 \%)$. Lifetime number of sexual partners was associated with HPV positivity. Condom use with both regular and sex-worker partners, and circumcision were inversely associated with HPV positivity [3]. Likewise, circumcision in another study was associated with reduced risk for detection of both oncogenic and non-oncogenic HPV types [4].

Even less is known about penile HPV infection in $\mathrm{HIV}$-positive men. The risk of penile cancer is elevated in HIV-positive men compared with the general population [5] but little is known about the natural history of penile disease in this population. A high prevalence of high-risk HPV types was present in the anus, penis and mouth $(78,36$ and $30 \%$, respectively) and was detected in a cohort of HIV-infected men without evidence of pathology in these areas [6].

\subsection{Diagnosis of HPV-associated lesions of the penis}

In addition to the problems associated with penile sampling, methods to diagnose penile lesions have been problematic. There is no standardized method to do so, but it is commonly accepted that magnification with application of acetic acid, similar to its use in the cervix, will result in improved diagnostic sensitivity, albeit at the expense of specificity [7,8]. Penile HPV infections may be latent, or may lead to subclinical or clinical disease. Latent infection is defined as the presence of HPV DNA within the tissue in the absence of any changes seen on visualization under magnification or histologic changes. A lesion, clinical or subclinical, is defined as the clinically visible and histologic modifications occurring due to HPV infection. Subclinical lesions are defined as those lesions visible only after application of acetic acid. Clinical lesions are defined as those visible to the naked eye without acetic acid.

The clinical examination should be carried out carefully on the entire anogenital region, including the penis, scrotum and perianal region with the help of a strong light and a magnifying lens (at least 4-fold). Subclinical lesions may be visualized after application of $5 \%$ acetic acid. While a colposcope may provide light and magnification, most acetowhite reactions will be detected by a hand-held magnifying lens.

The biological basis of acetowhitening is unknown. In lesions of external genitalia, areas with surface nuclear activity (parakeratosis) and/or increased cellular density will react. Acetic acid may be applied with a cotton ball mounted on a sponge-holding forceps, a scopette or by self-application of a gauze soaked in acetic acid. Acetic acid should be applied generously and the clinician must wait two to three minutes before beginning the examination. While recognized as a valuable aid to the diagnosis of penile disease, routine use of these techniques to examine at-risk men has not been advocated by professional organizations. In part, this reflects the absence of standardized training in this technique, limited specificity of acetowhitening and its unknown negative and positive predictive value in populations at different level of risk. Paradoxically, the areas of the penis that turn acetowhite the fastest are most often those with non-specific conditions such as inflammation and abrasion, and there is concern for over-treatment of non-HPV-related lesions.

Accurate identification may lead to better diagnosis of lesions and improved treatment, but the effect of treatment of penile disease has not been proven to reduce mortality among either men or women. Treatment of high-grade penile intraepithelial neoplasia may well reduce the incidence of penile cancer but this has not yet been demonstrated. Although condom use in male partners of women with CIN has been shown to 
increase regression of CIN [9], there are no data showing that treatment of HPV-associated disease reduces the risk of disease or infection in their female partners. For these reasons, routine screening of men with magnification and acetic acid has never become standard of practice.

With these caveats in mind, examination of men with these techniques may be useful under certain circumstances. These include identification of small, clinically inapparent or subclinical lesions in men who present to the clinician with genital warts. Men who present with symptoms such as itching or pain, or signs such as erythema must also be examined to rule out cancer or high-grade penile intraepithelial neoplasia.

Once identified, a penile biopsy may be needed to exclude the existence of penile cancer or penile intraepithelial neoplasia, or to confirm the diagnosis of HPVassociated lesions. Anesthesia, typically in the form of subcutaneous lidocaine is applied. A punch biopsy such as a Kevorkian, a $4 \mathrm{~mm}$ disposable Keyes punch, forceps and scissors, or a knife may be used. To use the Keyes punch, the clinician maintains the pressure and rotation until subcutaneous tissue is reached. The depth of the biopsy also depends on epidermal thickness. When the dermis is entered, there is a sensation of decreased resistance. The dermal tissue is cut with a small scalpel or scissors and the elliptical defect can be cauterized with Monsell's solution (ferric subsulfate) or sutured. Forceps can also be used to lift anesthetized skin or mucosa, and sharp scissors used to snip the biopsy.

\subsection{Histopathology of penile HPV-associated lesions}

Condyloma acuminata, commonly known as "warts", are exophytic, white grayish protuberances on keratinized skin, with a lobulated or irregular surface. Papillae may be prominent with finger-like projections that exhibit punctate and/or loop-like patterns visible with magnification unless the lesion is heavily keratinized. Some warts will be highly keratinized, thus appearing white. Their number varies from one to 50 or more lesions, and the sizes vary from 0.2 to $1.0 \mathrm{~cm}$, but they may become confluent involving large areas of genitalia. In uncircumcised men warts are most prevalent on the inner aspect of the prepuce and at the frenulum and coronal sulcus. In circumcised men the shaft is often involved. Warts may be present on the meatal lips of the urinary meatus or the distal area of the urethra up to $10-28 \%$ of men.
Condylomata acuminata must be distinguished from other papillomatous lesions in this area. On the penis, a variant of normal known as pearly penile papules may simulate warts [10]. These are hypertrphic papillae that form rows of discrete acuminate structures distributed circumferentially around the coronal sulcus. Reactive hyperplasia may also be associated with papillary structures in circumcised men. In these structures, the surface is the same as that of the surrounding skin and no vascular structures are detected. Condylomata lata of secondary syphilis should be distinguished from condylomata acuminata. Some warts may be pigmented, and in such cases may be difficult to distinguish from pigmented papules of high-grade penile intraepithelial neoplasia.

HPV types 6 and 11 are detected in $70-95 \%$ of condylomata acuminata, independent of the location, extent and duration of lesions. Oncogenic HPVs are not usually detected in typical warts in immunocompetent individuals.

HPV-associated lesions may also take the form of non-pigmented papules. These lesions have the color of normal skin or are occasionally brown and have a round or dome-shaped, slightly hyperkeratotic or smooth surface $[11,12]$. HPV-associated papules can be easily distinguished from the umbilicated pink-gray papules of molluscum contagiosum. Sebaceous glands of the prepuce should not be mistaken for HPV-associated papules. These are grey-yellow in color and magnification permits the identification of their structure [12].

Examination of the penis with acetic acid and magnification may also reveal subclinical macules consisting of smooth, flat, well demarcated acetowhite areas of normal epithelium with obvious capillary loops. They are usually found on mucosal surfaces such as the prepuce and glans and occasionally on the keratinized skin of the external genitalia. The differential diagnosis includes balanitis in which the acetowhitening is more diffuse, avascular and irregular; and yeast infection, in which multiple isolated acetowhite areas are often detected. In yeast infection capillaries are evident diffusely and are not restricted to the acetowhite areas. Other causes of acetowhitening includes traumatic microabrasions. While colposcopic examination often permits the clinician to distinguish subclinical HPVassociated lesions from infection or inflammation, it may not always be possible to do so, and local antiinflammatory treatment may be given to men prior to further work-up.

The term "Bowenoid papulosis" is used to describe papular or maculopapular lesions that show histolog- 


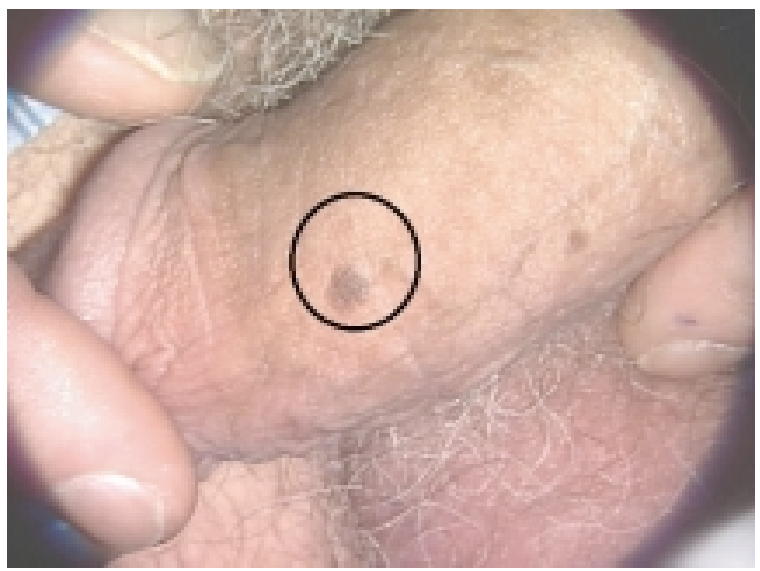

Fig. 1. Lesion with biopsy-confirmed penile intraepithelial neoplasia II-III. The lesion, shown in the black circle is flat and hyperpigmented

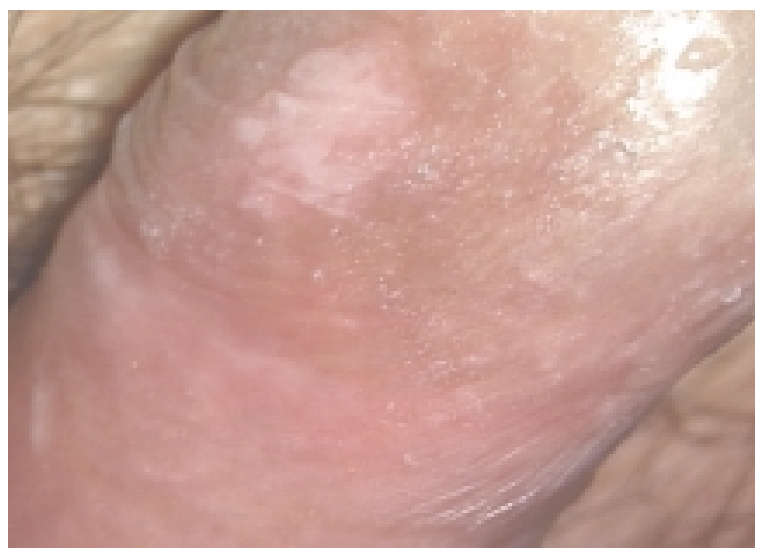

Fig. 2. Lesion with biopsy-confirmed penile intraepithelial neoplasia II. This lesion is from a different patient from that of Fig. 1, and shows a flat, well circumscribed, acetowhite lesion after application of $5 \%$ acetic acid.

ical features of high-grade intraepithelial neoplasia or carcinoma in situ $[13,14]$. These papules are usually hyperpigmented and are often flat despite the term "papulosis". They may be red or slightly brown with a smooth, glistening surface (Figs 1 and 2). They range in number from single lesions to about 30 and may coalesce to form plaques and they may exhibit strong acetowhitening. The differential diagnosis of Bowenoid papules includes pigmented warts, lentigo, naevi and melanoma, seborrheic warts, angiokeratomas, lichen planus and localized psoriatic lesions.

The histology of penile intraepithelial neoplasia in young adults shows features typical of other sites in the male and female genital tracts, including epithelial proliferation, hyperkeratosis and parakeratosis [15]. The cell nuclei are hyperchromatic, clumped and show loss of organization, maturation and cohesion. Mitotic figures are generally numerous and abnormal forms are often present. Potentially oncogenic HPV types are usually associated with these lesions and the most frequently identified type is HPV16 [14-16].

The prevalence of penile intraepithelial neoplasia is much lower in studies from the United States than in Europe. This may be linked to higher rates of circumcision, since circumcised male partners of women with CIN have a lower rate of penile intraepithelial neoplasia than uncircumcised men [17]. Circumcised men also have a lower prevalence of subclinical disease since many of these are often localized to the prepuce.

The natural history of high-grade penile intraepithelial neoplasia in young adults is not well known since natural history studies have not been done. It is likely however that conversion to invasive penile cancer is a rare event. In older patients, high-grade penile intraepithelial neoplasia mostly consists of Bowen's disease and Erythroplasia of Queyrat. These are believed to be precursor lesions of basaloid and warty carcinomas of the penis [18]. Consistent with the high prevalence of HPV in these lesions, their associated cancers have a high prevalence of HPV (Table 1). Precursors of predominantly HPV-negative keratinizing and verrucous penile carcinomas are not established.

Clinically, high-grade penile intraepithelial neoplasia in patients older than 50 years may be slightly elevated, plaque-like (Bowen's disease) or totally flat (Erythroplasia of Queyrat). Erythroplastic lesions appear as bright, velvet-red areas while the leucoplastic form appears as a white plaque, with a smooth or verrucous surface. Localized at first, these lesions may extend to large areas of external genitalia, including the perineum and perianal areas. These lesions may persist for long time and the risk of progression to cancer, although not precisely known, is higher than in penile intraepithelial neoplasia of young adults. The differential diagnosis includes lichen sclerosus, lichen simplex and psoriasis. Erosive or ulcerated areas and atypical vessels within the lesions are suggestive of invasion. Penile intraepithelial neoplasia in older patients is associated with the same HPV types found in intraepithelial neoplasia in younger patients [11].

Penile cancer is an uncommon cancer, but its incidence varies widely in different countries [19]. It is a rare cancer in North America, where circumcision is more common than in other regions of the world. Similar to vulvar cancer there may be two separate pathways to development of penile cancer. As described 
Table 1

Prevalence of HPV DNA using PCR in selected penile cancer case series ( $\geqslant 5$ cases)

\begin{tabular}{|c|c|c|c|c|}
\hline Reference & Study area & Detection method & Number of cases & $\begin{array}{l}\text { Overall HPV } \\
\text { positivity }(\%)\end{array}$ \\
\hline Rubin 2001 [69] & USA and South America & $\begin{array}{l}\text { PCR SPF } 10 \text { primers, LIPA } \\
\text { genotyping }\end{array}$ & $\begin{array}{l}106 \text { Keratinizing SCC } \\
5 \text { Warty SCC } \\
15 \text { basaloid SCC }\end{array}$ & $\begin{array}{l}34.9 \% \\
100 \% \\
80 \%\end{array}$ \\
\hline Levi 1998 [70] & South America & $\begin{array}{l}\text { PCR GH20/PCO } 4 \text { primers } \\
\text { MY09/MY11 PCR primers with } \\
\text { probing for }\end{array}$ & $\begin{array}{l}64 \\
50\end{array}$ & $\begin{array}{l}28 \% \\
56 \%\end{array}$ \\
\hline Carter 2001 [71] & USA & $\begin{array}{l}\text { PCR L1 consensus primers and type } \\
\text { specific primers for } 6 / 18\end{array}$ & 33 & $81.8 \%$ \\
\hline Cupp, 1995 [72] & USA & $\begin{array}{l}\text { MY09/MY } 11 \text { L1 primers and E6 } \\
\text { primers for } 16 / 18\end{array}$ & $\begin{array}{l}42 \text { squamous cell cancers } \\
13 \text { carcinoma in situ }\end{array}$ & $\begin{array}{l}54.8 \% \\
92.3 \%\end{array}$ \\
\hline Picconi, 2000 [73] & Argentina & $\begin{array}{l}\text { GP5/GP6 primers, typing by SCCP } \\
\text { analysis }\end{array}$ & 34 & $52.9 \%$ \\
\hline Bezerra, 2001 [74] & Brazil & $\mathrm{L} 1$ primer $\mathrm{PCR}$ & $\begin{array}{l}11 \text { warty carcinoma } \\
60 \text { SCC }\end{array}$ & $\begin{array}{l}45.5 \% \\
26.7 \%\end{array}$ \\
\hline Gregoire, 1995 [75] & USA and Paraguay & $\begin{array}{l}\text { PCR with specific priming for } \\
\text { HPV16 (E6), } 18(\mathrm{E} 6), 6 / 11(\mathrm{E} 1) \text {, al- } \\
\text { so E1 priming for wide range in- } \\
\text { cluding } 16,18,31,33,35,52\end{array}$ & $\begin{array}{l}45 \text { SCC } \\
12 \text { basaloid } \\
10 \text { papillary } \\
9 \text { warty } \\
6 \text { verrucous } \\
8 \text { Mixed SCC, other } \\
19 \text { Mixed SCC, warty or } \\
\text { basaloid }\end{array}$ & $\begin{array}{l}11.1 \% \\
75.0 \% \\
0 \% \\
22.2 \% \\
0 \% \\
0 \% \\
47.4 \%\end{array}$ \\
\hline
\end{tabular}

above, basaloid and warty penile cancers are frequently HPV-associated (about $80-100 \%$ ), in contrast to a smaller proportion of keratinizing and verrucous penile carcinomas (Table 1). Overall, about $50 \%$ of penile cancers contain HPV DNA. HPV16 is the most common HPV type detected in penile cancer. HPV 31 or 33 were described only rarely. Giant condylomata acuminata, also known as Buschke-Lowenstein tumors, may precede the development of verrucous carcinomas in some cases and may contain HPV6 or 11.

\subsection{Treatment of HPV-associated penile disease}

The definitive diagnosis of HPV-related penile disease requires a biopsy, but this is usually unnecessary. Typical warts, papillomas and non-pigmented papules do not need to be biopsied. For subclinical lesions, in case of doubt, local anti-inflammatory treatment should be given in an effort to induce regression of non-specific acetowhite areas. Lesions with features suggesting intraepithelial neoplasia should be biopsied to confirm the diagnosis and to exclude the existence of invasive cancer. This is particularly true in patients older than 40 , since the risk of progression to invasion of intraepithelial neoplasia is higher in this group.

There is general consensus about the need for treatment of clinically detectable warts and penile intraepithelial neoplasia. There is less consensus on the need for treatment of subclinical lesions. In choosing the treatment modality, factors to consider are differences in size, location, number and histology of the lesions [20]. Other factors to consider include whether the lesions are newly diagnosed or recurrent, and the patient's compliance and pain threshold. Small warts may be treated topically. Patient-applied therapies such as podophyllotoxin or imiquimod cream are useful first approaches. They have the advantage of allowing patients to treat themselves, but have the disadvantages of requiring several weeks or more for therapeutic responses. Patients must also be able to visualize the lesions to know where to apply the creams. Topical treatment with $80 \%$ trichloracetic acid (TCA) or liquid nitrogen are acceptable options but must be applied by the treating clinician. This may be preferable for lesions that are too small or inaccessible for self-treatment by the patient, and have the advantage of inducing a therapeutic response quickly. Use of TCA is preferable on non-keratinized mucosal lesions, since liquid nitrogen is painful at those locations. Liquid nitrogen is preferred by some clinicians for cutaneous and hyperkeratinized mucosal lesions. Both treatments often require multiple applications by clinicians, typically 1-2 weeks apart. Second-line therapies that can be used include interferon, 5 fluorouracil, electrocautery and laser surgery. Lesions persisting after local treatment should be considered for surgical or laser ablation. 
Treatment of penile intraepithelial neoplasia should be performed after biopsies have excluded invasive penile cancer. The choice of ablative therapy will depend on the size and location of the lesions. A physician may remove it with laser vaporisation or electrosurgery. These lesions can also be treated with TCA and liquid nitrogen. Treatment with podophyllotoxin or imiquimod isn't currently recommended as these drugs have only been approved for treatment of genital warts.

As with all cancers, penile cancer is treated by stage, type, the patient's age and overall condition. For Stage I (localized cancer) the cure rate is high and may involve wide local excision. If the tumor begins in the glans and involves other tissues, treatment may involve partial penectomy and lymph node removal, external radiation therapy and microsurgery. In Stage II, cancer cells have spread to the glans and shaft of the penis, and surgery involving partial, total, or radical penectomy is usually the first approach, or radiation therapy followed by penectomy. Stage III involves cancer that has spread beyond the penis. In addition to penectomy, lymph node removal on both sides of the groin, radiation therapy and chemotherapy may be required. A variety of different chemotherapy drugs have been used in penile cancer in combination with radiation therapy. For stage IV, the cancer has metastasized to other parts of the body. Penectomy, wide local excision, and microsurgery may be used as well as chemotherapy and radiation therapy.

\subsection{Relationship between penile HPV infection and $H P V$-associated disease in women}

Since the penis is the presumed source of most HPV infection that is transmitted to women, there has been increasing interest in determining concordance of HPV types between sexual partners. Consistent with a relationship between penile and cervical HPV infection, male condom use has been shown to promote regression of human papillomavirus-associated penile lesions in male sexual partners of women with cervical intraepithelial neoplasia [9], and male circumcision is associated with a reduced risk of cervical cancer in their current female partners [21]. Consistent condom use has recently been shown to reduce the risk of transmission by about $70 \%$ [22].

In one study of women with CIN, penile lesions were seen in $68 \%$ of male partners, and most lesions were flat and subclinical. HPV was detected in $59 \%$ of the penile scrapings and contained mainly oncogenic HPV types [23]. However the degree of concordance of HPV types between sexual partners has varied considerably between different studies. In one study of husbands of women with invasive cervical carcinoma or cervical carcinoma in situ, $18 \%$ of the husbands of women with invasive cervical carcinoma, and $21 \%$ of the husbands of women with cervical carcinoma in situ were positive for penile HPV DNA, and concordance of HPV types was low between husband and wife [24]. In a more recent study, among couples in which both partners were HPV-positive, $57.8 \%$ of the men had the same HPV type as their partners [25]. In another study of male partners of women positive for HPV using the HCII method, using HCII on penile brushings, $32 \%$ were positive for one or more HPV types in the high-risk HPV group, $14 \%$ in the low-risk HPV group and $24 \%$ in both groups [2]. Using PCR in a study of university students in South Korea, HPV DNA was detected more frequently in female students $(15.2 \%)$ than in male students $(8.7 \%)$. Among those students who reported being sexually active, the prevalence of HPV was $38.8 \%$ in females and $10.6 \%$ in males [26]. In contrast, in a study of Mexican men attending vasectomy clinics, the prevalence of HPV in Mexican men was similar to that of Mexican women of the same age [3].

\section{HPV infection and disease of the anus in men}

\subsection{Epidemiology of anal HPV infection and $H P V$-associated anal neoplasia}

Anal HPV infection is remarkably common in men who have sex with men (MSM) and HIV-positive men regardless of sexual orientation [27]. HPV infection is most common in HIV-positive MSM [27,28]. Almost all HIV-positive MSM have anal HPV infection, frequently with multiple HPV types. HIV-positive heterosexual men also have a high prevalence of anal HPV infection [29], as do HIV-negative MSM [27].

Anal HPV infection is the most significant risk factor for the development of anal intraepithelial neoplasia (AIN), the precursor lesion to anal cancer. Consistent with their high prevalence of anal HPV infection, the prevalence of AIN in men is highest in HIV-positive MSM [30] followed by HIV-negative MSM [27,31] and HIV-positive heterosexual men [29]. The incidence of high-grade AIN is also high both HIV-positive and HIVnegative MSM [32-35]. Among HIV-positive MSM this has also been observed despite the introduction of highly active antiretroviral therapy (HAART) [36,37]. In HIV-positive men, anal HPV infection and lower 
Table 2

Prevalence of HPV DNA using PCR in selected anal cancer case series ( $\geqslant 5$ cases)

\begin{tabular}{|c|c|c|c|c|}
\hline Reference & Study area & Detection method & Number of cases & $\begin{array}{c}\text { Overall HPV } \\
\text { positivity (\%) }\end{array}$ \\
\hline Carter J 2001 [71] & USA & PCR L1 consensus primers & 64 & $93.8 \%$ \\
\hline Frisch, 1999 [76] & Denmark and Sweden & GP5+/GP6+ & 331 & $\begin{array}{l}83.7 \% \text { for HR group } \\
4 \% \text { for low-risk group }\end{array}$ \\
\hline Daling, 2004 [43] & USA & MY09/MY11 PCR & $\begin{array}{l}\frac{119 \text { men }}{81 \text { SCC }} \\
6 \text { Cloacogenic /basaloid } \\
8 \text { Adenocarcinoma } \\
\frac{187 \text { women }}{98 \text { SCC }} \\
35 \text { Cloacogenic /basaloid } \\
12 \text { Adenocarcinoma }\end{array}$ & $\begin{array}{l}92.6 \% \\
100 \% \\
25 \% \\
91.8 \% \\
97.1 \% \\
50.0 \%\end{array}$ \\
\hline Poletti, 1998 [77] & Switzerland & $\begin{array}{l}\text { PCR with PU-1M primers for } \\
16 / 18 / 31 / 33 / 52 / 58 \text { and PU-31B } \\
\text { primers for } 6 / 11\end{array}$ & 33 & $39.4 \%$ \\
\hline Frisch, 1997 [46] & Sweden and Denmark & GP5+/GP6+ primers & 388 & $87.6 \%$ \\
\hline Shroyer, 1995 [78] & USA & MY0/MY11 primers & $\begin{array}{l}11 \text { basaloid cancer } \\
16 \text { non-basaloid cancers }\end{array}$ & $\begin{array}{l}90.9 \% \\
75.0 \%\end{array}$ \\
\hline
\end{tabular}

CD4+ cell count are the main risk factors associated with prevalent and incident AIN [30]. In a study of AIN in HIV-negative MSM, risk factors included number of receptive anal sex partners and injection drug use, but HPV infection, and a higher number of HPV types detected were the strongest risk factors for AIN [31]. Similar risk factors were found in an earlier study [30].

Despite evidence that immunosuppression as measured by CD4+ level is linked to the development of AIN, the role of immunosuppression in the progression from high-grade AIN to invasive cancer is unclear. Unlike other HIV-related malignancies such as Kaposi's sarcoma and non-Hodgkin's lymphoma, there is no significant increase in anal cancer after an AIDS diagnosis [38]. Other factors such as host genomic mutations, rather than intrinsic immunosuppression, may be more important in progression to invasive cancer [39].

Like cervical cancer, anal cancer is strongly associated with HPV infection [40] (Table 2). While less common than cervical cancer, anal cancer is increasing in incidence in both women and men, from about 10 cases per million to 20 cases per million from 1973 to 2000 [41]. Some of this increase is attributable to a rising incidence among MSM. Before the HIV epidemic, it was estimated that the incidence of anal cancer among MSM was estimated to be as high as 37 per 100,000 person years, comparable to the incidence of invasive cervical cancer in women prior to the introduction of cervical cytology screening [42].

Similar to cervical cancer, HPV infection is one of the most important risk factors for anal cancer and
HPV16 is the most common HPV type isolated in anal cancer [43]. Sexual activity was shown in early studies to be a risk factor for anal cancer [43-45]. In a Scandinavian study, independent risk factors for anal cancer in men included higher numbers of sexual partners and a history of other sexually transmitted infections [46]. Cigarette smoking was a risk factor for anal cancer in at least three studies $[43,45,47]$.

Immunocompromise is another key risk factor, particularly HIV-associated immunocompromise. Data from matching cancer and AIDS registries show that the relative risk of invasive anal cancer was 37 in HIV-positive MSM compared with the general population [5], and the incidence of anal cancer among HIVpositive MSM is estimated to be double that of HIVnegative MSM. Consistent with the data on AIN and HAART, recent data also show that the incidence of anal cancer is continuing to increase in HIV-positive MSM since the introduction of HAART [48-50]. Other immunocompromised patients such as transplant recipients are also at increased risk of anal cancer [51-54].

\subsection{Diagnosis of anal HPV-associated anal neoplasia}

Given the similarity between anal and cervical cancer, and because cervical Pap testing has been successful in cervical cancer screening, Palefsky and others have proposed an anal cancer screening program that incorporates many of the principles of cervical cancer screening [55]. Anal cancer screening is recommended 
for populations at high risk of anal cancer. These include all HIV-positive women and men, MSM, women with a history of vulvar or cervical cancer and organ transplant recipients. Anal cytology testing every year to two years has been projected to be a cost-effective intervention to prevent anal cancer in HIV-positive [56] and HIV-negative MSM [57].

Abnormal anal cytology should be followed with measures to diagnose anal disease histologically and to exclude anal cancer. All patients at risk for anal cancer should also have a digital rectal examination to detect palpable lesions consistent with cancer. Lesions are typically visualized using high-resolution anoscopy (HRA). HRA is similar to cervical colposcopy and uses identical equipment (a powerful light source and binocular lenses). Using HRA, lesions that have contributed to abnormal cytologic findings can be visualized and biopsied. $3 \%$ acetic acid can be used to assist in the visualization of abnormal tissue, similar to the changes induced by acetic acid in the cervix and on the penis. HPV-infected lesions turn white relative to surrounding normal tissue. Other signs of AIN include abnormal vasculature with features such as punctation, mosaicism and atypical vessels [58] (Fig. 3). The application of Lugol's (iodine) solution can also increase specificity. Abnormal tissue remains unstained or appears light yellow, unlike adjacent normal tissue which stains dark brown or mahogany due to the presence of glycogen in the cells.

The sensitivity of anal cytology to detect biopsy proven AIN is 50 to $80 \%$ [59], similar to the sensitivity of cervical Pap tests to detect biopsy-proven CIN. Anal cytology is more sensitive in HIV-positive men compared with HIV-negative men in detecting AIN, most likely reflecting the presence of more extensive HIV-associated disease in the HIV-positive population.

\subsection{Histopathology of anal intraepithelial neoplasia}

Similar to the classification system used for cervical cytology, anal cytology is classified as normal, atypical squamous cells of undetermined significance (ASCUS), atypical squamous cells- cannot rule out highgrade disease (ASC-H), low-grade squamous intraepithelial lesions (LSIL), high-grade squamous intraepithelial lesions (HSIL) and invasive carcinoma. The histologic correlate of CIN is AIN, which is a precursor to anal cancer [60-62]. The grading system reflects the degree of histologic abnormality observed. AIN I is not likely to progress to anal cancer whereas AIN II and AIN III are believed to be the true anal cancer pre-

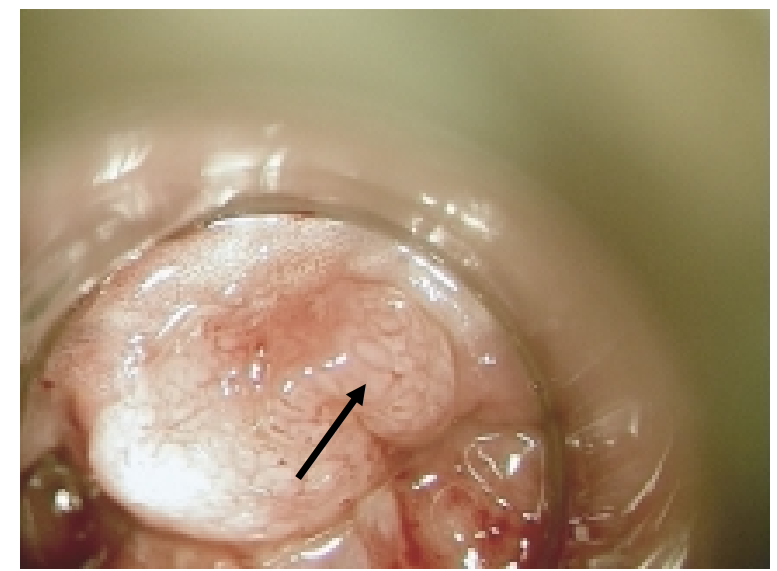

Fig. 3. Lesion with biopsy-confirmed anal intraepithelial neoplasia III. Flat, plaque-like lesion in the anal canal is shown with a black arrow and demonstrates atypical blood vessels.

cursors. Like CIN 1, AIN 1 shows signs of replicative HPV infection, including koilocytosis and few signs of cellular atypia. AIN II and AIN III, like their cervical counterparts, show progressively increasing proliferation and replacement of the normal epithelium by cells with a basal morphology and large nuclear-cytoplasmic ratios, and mitoses in the more superficial cell layers.

\subsection{Treatment of HPV-associated anal neoplasia}

\subsubsection{AIN and condyloma}

Because of anatomical challenges such as hemorrhoids and crypts, it can be more difficult to treat AIN compared with CIN. Recent guidelines were published for the treatment of AIN in the setting of HIV infection [55,63]. As in cervical disease, histology, lesion size, lesion location and HIV-status are the main determinants that may influence treatment modalities and treatment goals. While AIN I likely does not progress directly to cancer, treatment may of AIN 1 reduce the risk of enlargement which may preclude the use of topical agents, decrease the risk of progression to AIN II or AIN III, and may provide symptomatic or psychological relief. AIN II and AIN III should be treated whenever possible to prevent cancer. Some experts have a lower threshold to treat HIV-positive men with minimal AIN I in HIV-positive men since there is a faster rate of progression to AIN II or AIN III compared with HIV-negative men [35].

Smaller lesions are generally easier to treat than larger lesions. Topical therapy can be used for relatively flat intra-anal (85\% trichloroacetic acid, liquid nitrogen) or perianal lesions (imiquimod, podophyllotoxin, 
$85 \%$ trichloroacetic acid, liquid nitrogen) smaller than $1 \mathrm{~cm}^{2}$ at the base. Large circumferential intra-anal lesions, particularly in HIV-positive men, have a high recurrence rate if removed rate [64]. Because treatment of extensive intra-anal lesions may require multiple staged procedures and may be associated with a high morbidity (post-operative pain), and high recurrence rate these individuals may be followed closely without treatment. Large, circumferential intra-anal lesions may be removed surgically if patients are symptomatic or if the goal is to rule out anal cancer. Lesions in the middle-sized category (too big for topical therapy, but not large enough to require surgery) are increasingly being treated with infrared coagulation (Redfield Corporation, Rochelle Park, New Jersey) which has been licensed for treatment of condylomata acuminata and tattoo removal [65]. After local anesthesia with $1 \%$ lidocaine with epinephrine, the infrared coagulator is used to destroy the lesion by generating intense heat at the tip of the probe. Coagulative necrosis occurs without a smoke plume. This procedure is well tolerated with fewer complications compared with surgery, with only mild to moderate post-procedure discomfort occurring for a few days. Other therapies for AIN may have promise, including photodynbamic therapy [66] and intra-anal imiquimod [67], but these require further study.

Perianal condyloma may be amenable to local patient-applied therapy such as imiquimod or podophyllotoxin, which are not recommended for intra-anal use. Liquid nitrogen, electrocautery, TCA and IRC may be used in the office by the clinician. Condyloma that are too large for office-based therapy usually require removal in the surgical suite. Perianal high-grade disease may be treated with TCA, IRC or surgery.

\subsubsection{Anal cancer}

Treatment of anal cancer depends on the stage of the cancer and the patient's age and general health. Stage 0 , also classified as anal carcinoma in situ, can be removed locally, similar to the treatment described above for AIN II-III. Stage I cancer, which is smaller than two centimeters in diameter, but has not spread to the muscle tissue, can sometimes be treated by local resection, but the usual therapy is a combination of chemotherapy with radiation therapy. If cancer recurs, abdominoperineal resection may remove the remaining cancer. In Stage II cancer, which is larger than two centimeters in diameter, but has not spread to nearby organs or lymph nodes, the treatment options are the same as for Stage I. For stage III-A cancer, which has spread to the lymph nodes around the rectum or to nearby organs such as the bladder, radiation plus chemotherapy is still an option, but because the cancer has spread to the lymph nodes, surgery may be needed to remove parts of the rectum or colon as well as to perform a lymph node dissection. For Stage III-B cancer, in which the cancer has spread to both lymph nodes and nearby organs, radiation and chemotherapy, followed by surgery is the best combination. The recovery rate from Stage IV metastatic anal cancer is low and most treatments are palliative (designed to relieve symptoms rather than cure the cancer).

The standard of care for the treatment of all but Stage IV invasive anal cancer is thus combined modality therapy (CMT) with both RT and chemotherapy (5-FU and mitomycin used together), with or without surgery as described above. More recently, cis-platinum has been increasingly used in place of mitomycin [68]. CMT avoids the morbidity of abdominoperineal resection (APR) which involves removal of the anorectum and necessitates a permanent colostomy. CMT has also been successfully employed in HIV-positive men [68], including those with low CD4+ level but who have a well-controlled HIV viral load on HAART.

\section{Conclusions}

The consequences of anogenital HPV infection in men must be measured both in terms of their ability to spread HPV infection to their sexual partners, as well as the lesions, including cancers, that affect men directly. Although they share much in common with cervical HPV infection and disease, the natural history of penile and anal HPV infection, intraepithelial neoplasia and cancer is not well understood. Factors that lead to transmission of HPV infection between sexual partners requires further study, as will the effect of prophylactic HPV vaccination of women on male disease. Conversely, prophylactic HPV vaccination of men needs to be studied for its effect on reducing the burden of male disease and protecting the health of women by reducing their exposure to HPV and improving their "herd immunity". As a result of the success of the HPV vaccine in women, more attention than ever is currently being given to the "male factor", and it is expected that these studies will yield important information that will be valuable to the treatment and control of HPV-associated neoplasia in both women and men. 


\section{Acknowledgements}

The author gratefully acknowledges Ms. Victoria Kuohung and Dr. Michael Berry for the photos in Figs 1 to 3 .

\section{References}

[1] S.B. Baldwin, D.R. Wallace and M.R. Papenfuss, Human papillomavirus infection in men attending a sexually transmitted disease clinic, J Infect Dis 187 (2003), 1064-1070.

[2] S.M. Nicolau, C.G. Camargo and J.N. Stavale, Human papillomavirus DNA detection in male sexual partners of women with genital human papillomavirus infection, Urology $\mathbf{6 5}$ (2005), 251-255.

[3] S. Vaccarella, E. Lazcano-Ponce and J.A. Castro-Garduno, Prevalence and determinants of human papillomavirus infection in men attending vasectomy clinics in Mexico, Int J Cancer 119 (2006), 1934-1939.

[4] S.B. Baldwin, D.R. Wallace, M.R. Papenfuss, M. Abrahamsen, L.C. Vaught and A.R. Giuliano, Condom use and other factors affecting penile human papillomavirus detection in men attending a sexually transmitted disease clinic, Sex Transm Dis 31 (2004), 601-607.

[5] M. Frisch, R.J. Biggar and J.J. Goedert, Human papillomavirus-associated cancers in patients with human immunodeficiency virus infection and acquired immunodeficiency syndrome, J Natl Cancer Inst 92 (2000), 1500-1510.

[6] G. Sirera, S. Videla and M. Pinol, High prevalence of human papillomavirus infection in the anus, penis and mouth in HIVpositive men, AIDS 20 (2006), 1201-1204

[7] R. Barrasso, J. De Brux, O. Croissant and G. Orth, High prevalence of papillomavirus-associated penile intraepithelial neoplasia in sexual partners of women with cervical intraepithelial neoplasia, N Engl J Med 317 (1987), 916-923.

[8] J.M. Palefsky and R. Barrasso, HPV infection and disease in men, Obstet Gynecol Clin North Am 23 (1996), 895-916.

[9] M.C. Bleeker, C.J. Hogewoning and F.J. Voorhorst, Condom use promotes regression of human papillomavirus-associated penile lesions in male sexual partners of women with cervical intraepithelial neoplasia, Int J Cancer 107 (2003), 804-810.

[10] A.B. Ackerman and R. Kronberg, Pearly penile papules. Acral angiofibromas, Arch Dermatol 108 (1973), 673-675.

[11] R. Barrasso and S. Jablonska, Clinical, colposcopic and histologic spectrum of male human papillomavirus-associated genital lesions, Clin Pract Gynecol 2 (1989), 73-101.

[12] J. Palefsky and R. Barasso, HPV infection and disease in men, Obstet Gyn Clin North Am 23 (1996), 895-916.

[13] T.R. Wade, A.W. Kopf and A.B. Ackerman, Bowenoid papulosis of the penis, Cancer 42 (1978), 1890-1903.

[14] S. Obalek, S. Jablonska and G. Orth, HPV-associated intraepithelial neoplasia of external genitalia, Clin Dermatol 3 (1985), 104-113.

[15] S. Obalek, S. Jablonska, S. Beaudenon, L. Walczak and G. Orth, Bowenoid papulosis of the male and female genitalia: risk of cervical neoplasia, J Am Acad Dermatol 14 (1986), 433-444.

[16] H. Ikenberg, L. Gissmann, G. Gross, E.I. Grussendorf-Conen and $\mathrm{H}$. zur Hausen, Human papillomavirus type-16-related DNA in genital Bowen's disease and in Bowenoid papulosis, Int J Cancer 32 (1983), 563-565.
[17] R.U. Levine, C.P. Crum, E. Herman, D. Silvers, A. Ferenczy and R.M. Richart, Cervical papillomavirus infection and intraepithelial neoplasia: a study of male sexual partners, Obstet Gynecol 64 (1984), 16-20.

[18] G. Gross and H. Pfister, Role of human papillomavirus in penile cancer, penile intraepithelial squamous cell neoplasias and in genital warts, Med Microbiol Immunol (Berlin) 193 (2004), 35-44.

[19] D.M. Parkin, S.L. Whelan, J. Ferlay and H. Storm, Cancer Incidence in Five Continents, IARC CancerBase Lyon No. 7 (2005), I-VIII.

[20] Centers for Disease Control and Prevention. Sexually transmitted diseases treatment guidelines 2002, MMWR 51 (2002), RR-6.

[21] X. Castellsague, F.X. Bosch and N. Munoz, Male circumcision, penile human papillomavirus infection, and cervical cancer in female partners, $N$ Engl J Med 346 (2002), 1105-1112.

[22] R.L. Winer, J.P. Hughes and Q. Feng, Condom use and the risk of genital human papillomavirus infection in young women, $N$ Engl J Med 354 (2006), 2645-2654.

[23] M.C. Bleeker, C.J. Hogewoning and A.J. Van Den Brule, Penile lesions and human papillomavirus in male sexual partners of women with cervical intraepithelial neoplasia, J Am Acad Dermatol 47 (2002), 351-357.

[24] S. Franceschi, X. Castellsague and L. Dal Maso, Prevalence and determinants of human papillomavirus genital infection in men, Br J Cancer 86 (2002), 705-711.

[25] M.C. Bleeker, C.J. Hogewoning and J. Berkhof, Concordance of specific human papillomavirus types in sex partners is more prevalent than would be expected by chance and is associated with increased viral loads, Clin Infect Dis 41 (2005), 612-620.

[26] H.R. Shin, S. Franceschi and S. Vaccarella, Prevalence and determinants of genital infection with papillomavirus, in female and male university students in Busan, South Korea, $J$ Infect Dis 190 (2004), 468-476.

[27] J.M. Palefsky, E.A. Holly, M.L. Ralston, M. Da Costa and R.M. Greenblatt, Prevalence and risk factors for anal human papillomavirus infection in human immunodeficiency virus (HIV)-positive and high-risk HIV-negative women, J Infect Dis 183 (2001), 383-391.

[28] N. Kiviat, A. Rompalo and R. Bowden, Anal human papillomavirus infection among human immunodeficiency virusseropositive and -seronegative men, J Infect Dis 162 (1990), 358-361.

[29] C. Piketty, T.M. Darragh and M. Da Costa, High prevalence of anal human papillomavirus infection and anal cancer precursors among HIV-infected persons in the absence of anal intercourse, Ann Intern Med 138 (2003), 453-459.

[30] J.M. Palefsky, E.A. Holly and M.L. Ralston, Anal squamous intraepithelial lesions in HIV-positive and HIV-negative homosexual and bisexual men: prevalence and risk factors, $J$ Acquir Immune Defic Syndr Hum Retrovirol 17 (1998), 320-326.

[31] P.V. Chin-Hong, E. Vittinghoff and R.D. Cranston, Agerelated prevalence of anal cancer precursors in homosexual men: the EXPLORE study, J Natl Cancer Inst 97 (2005), 896-905.

[32] C.W. Critchlow, C.M. Surawicz, K.K. Holmes et al., Prospective study of high grade anal squamous intraepithelial neoplasia in a cohort of homosexual men: influence of HIV infection, immunosuppression and human papillomavirus infection, AIDS 9 (1995), 1255-1262.

[33] C. Piketty, T.M. Darragh and I. Heard, High prevalence of anal squamous intraepithelial lesions in HIV-positive men despite 
the use of highly active antiretroviral therapy, Sex Transm Dis 31 (2004), 96-99.

[34] J.M. Palefsky, E.A. Holly, M.L. Ralston, N. Jay, J.M. Berry and T.M. Darragh, High incidence of anal high-grade squamous intraepithelial lesions among HIV-positive and HIVnegative homosexual/bisexual men, AIDS 12 (1998), 495-503.

[35] J.M. Palefsky, E.A. Holly and C.J. Hogeboom, Virologic, immunologic, and clinical parameters in the incidence and progression of anal squamous intraepithelial lesions in HIVpositive and HIV-negative homosexual men, J Acquir Immune Defic Syndr Hum Retrovirol 17 (1998), 314-319.

[36] J.M. Palefsky, E.A. Holly and M.L. Ralston, Effect of highly active antiretroviral therapy on the natural history of anal squamous intraepithelial lesions and anal human papillomavirus infection, J Acquir Immune Defic Syndr 28 (2001), 422-428.

[37] J.M. Palefsky, E.A. Holly and J.T. E, Anal intraepithelial neoplasia in the HAART era among HIV-positive men who have sex with men, AIDS 19 (2005), 1407-1414.

[38] M. Frisch, R.J. Biggar, E.A. Engels and J.J. Goedert, Association of cancer with AIDS-related immunosuppression in adults, JAMA 285 (2001), 1736-1745.

[39] S.E. Gagne, R. Jensen and A. Polvi, High-resolution analysis of genomic alterations and human papillomavirus integration in anal intraepithelial neoplasia, J Acquir Immune Defic Syndr 40 (2005), 182-189.

[40] D.M. Parkin, The global health burden of infection-associated cancers in the year 2002, Int J Cancer (2006).

[41] L.G. Johnson, M.M. Madeleine, L.M. Newcomer, S.M. Schwartz and J.R. Daling, Anal cancer incidence and survival: the surveillance, epidemiology, and end results experience, 1973-2000, Cancer 101 (2004), 281-288.

[42] J.R. Qualters, N.C. Lee, R.A. Smith and R.E. Aubert, Breast and cervical cancer surveillance, United States, 1973-1987, MMWR 41 (1992), 1-15.

[43] J.R. Daling, M.M. Madeleine and L.G. Johnson, Human papillomavirus, smoking, and sexual practices in the etiology of anal cancer, Cancer 101 (2004), 270-280.

[44] J.R. Daling, N.S. Weiss and T.G. Hislop, Sexual practices, sexually transmitted diseases, and the incidence of anal cancer, N Engl J Med 317 (1987), 973-977.

[45] E.A. Holly, A.S. Whittemore, D.A. Aston, D.K. Ahn, B.J. Nickoloff and J.J. Kristiansen, Anal cancer incidence: genital warts, anal fissure or fistula, hemorrhoids, and smoking, J Natl Cancer Inst 81 (1989), 1726-1731.

[46] M. Frisch, B. Glimelius and A.J. van den Brule, Sexually transmitted infection as a cause of anal cancer, $N$ Engl J Med 337 (1997), 1350-1358.

[47] J.R. Daling, K.J. Sherman and T.G. Hislop, Cigarette smoking and the risk of anogenital cancer, Am J Epidemiol 135 (1992), 180-189.

[48] M. Bower, T. Powles and T. Newsom-Davis, HIV-Associated Anal Cancer: Has Highly Active Antiretroviral Therapy Reduced the Incidence or Improved the Outcome? J Acquir Immune Defic Syndr 37 (2004), 1563-1565.

[49] E.Y. Chiao, S.E. Krown, E.A. Stier and D. Schrag, A population-based analysis of temporal trends in the incidence of squamous anal canal cancer in relation to the HIV epidemic, $J$ Acquir Immune Defic Syndr 40 (2005), 451-455.

[50] M. Bower, C. Palmieri and T. Dhillon, AIDS-related malignancies: changing epidemiology and the impact of highly active antiretroviral therapy, Curr Opin Infect Dis 19 (2006), 14-19.

[51] I. Penn, Cancer in the immunosuppressed organ recipient, Transplant Proc 23 (1991), 1771-1772.
[52] I. Penn, The changing pattern of posttransplant malignancies, Transplant Proc 23 (1991), 1101-1103.

[53] J. Adami, H. Gabel and B. Lindelof, Cancer risk following organ transplantation: a nationwide cohort study in Sweden, Br J Cancer 89 (2003), 1221-1227.

[54] H.S. Patel, A.R. Silver and J.M. Northover, Anal cancer in renal transplant patients, Int J Colorectal Dis (2005), 1-5.

[55] P.V. Chin-Hong and J.M. Palefsky, Natural history and clinical management of anal human papillomavirus disease in men and women infected with human immunodeficiency virus, Clin Infect Dis 35 (2002), 1127-1134.

[56] S.J. Goldie, K.M. Kuntz, M.C. Weinstein, K.A. Freedberg, M.L. Welton and J.M. Palefsky, The clinical effectiveness and cost-effectiveness of screening for anal squamous intraepithelial lesions in homosexual and bisexual HIV-positive men, JAMA 281 (1999), 1822-1829.

[57] S.J. Goldie, K.M. Kuntz, M.C. Weinstein, K.A. Freedberg and J.M. Palefsky, Cost-effectiveness of screening for anal squamous intraepithelial lesions and anal cancer in human immunodeficiency virus-negative homosexual and bisexual men, $A m$ J Med 108 (2000), 634-641.

[58] N. Jay, E.A. Holly, M. Berry, C.J. Hogeboom, T.M. Darragh and J.M. Palefsky, Colposcopic correlates of anal squamous intraepithelial lesions, Dis Col Rectum 40 (1997), 919-928.

[59] J.M. Palefsky, E.A. Holly, C.J. Hogeboom, N. Jay, M. Berry and T.M. Darragh, Anal cytology as a screening tool for anal squamous intraepithelial lesions, J Acq Immun Defic Syndr 14 (1997), 415-422.

[60] C. Fenger and V.T. Nielsen, Precancerous changes in the anal canal epithelium in resection specimens, Acta Pathol Microbiol Immunol Scand [a] 94 (1986), 63-69.

[61] T.E. Marfing, M.E. Abel and D.M. Gallagher, Perianal Bowen's disease and associated malignancies. Results of a survey, Dis Colon Rectum 30 (1987), 782-785.

[62] A.B. Jaeger, A. Gramkow, H. Hjalgrim, M. Melbye and M. Frisch, Bowen disease and risk of subsequent malignant neoplasms: a population-based cohort study of 1147 patients, Arch Dermatol 135 (1999), 790-793.

[63] C.A. Benson, J.E. Kaplan, H. Masur, A. Pau and K.K. Holmes, Treating opportunistic infections among HIV-exposed and infected children: recommendations from CDC, the National Institutes of Health, and the Infectious Diseases Society of America, MMWR Recomm Rep 53 (2004), 1-112.

[64] G.J. Chang, J.M. Berry, N. Jay, J.M. Palefsky and M.L. Welton, Surgical treatment of high-grade anal squamous intraepithelial lesions: a prospective study, Dis Colon Rectum 45 (2002), $453-458$.

[65] S.E. Goldstone, A.Z. Kawalek and J.W. Huyett, Infrared coagulator: a useful tool for treating anal squamous intraepithelial lesions, Dis Colon Rectum 48 (2005), 1042-1054.

[66] J.H. Scholefield, Treatment of grade III anal intraepithelial neoplasia with photodynamic therapy: report of a case, Dis Colon Rectum 46(11) (2003), 1555-1559.

[67] A. Kreuter, B. Hochdorfer and M. Stucker, Treatment of anal intraepithelial neoplasia in patients with acquired HIV with imiquimod 5\% cream, J Am Acad Dermatol 50 (2004), 980981.

[68] J.M. Berry, J.M. Palefsky and M.L. Welton, Anal cancer and its precursors in HIV-positive patients: perspectives and management, Surg Oncol Clin N Am 13 (2004), 355-373.

[69] M.A. Rubin, B. Kleter and M. Zhou, Detection and typing of human papillomavirus DNA in penile carcinoma: evidence for multiple independent pathways of penile carcinogenesis, Am J Pathol 159 (2001), 1211-1218. 
[70] J.E. Levi, P. Rahal, A.S. Sarkis and L. Villa, Human papillomavirus DNA and p53 status in penile carcinomas, Int $J$ Cancer 76 (1998), 779-783.

[71] J.J. Carter, M.M. Madeleine and K. Shera, Human papillomavirus 16 and $18 \mathrm{~L} 1$ serology compared across anogenital cancer sites, Cancer Res 61 (2001), 1934-1940.

[72] M.R. Cupp, R.S. Malek, J.R. Goellner, T.F. Smith and M.J. Espy, The detection of human papillomavirus deoxyribonucleic acid in intraepithelial, in situ, verrucous and invasive carcinoma of the penis, J Urol 154 (1995), 1024-1029.

[73] M.A. Picconi, A.M. Eijan and A.L. Distefano, Human papillomavirus (HPV) DNA in penile carcinomas in Argentina: analysis of primary tumors and lymph nodes, J Med Virol 61 (2000), 65-69.

[74] A.L. Bezerra, A. Lopes, G. Landman, G.N. Alencar, H. Torloni and L.L. Villa, Clinicopathologic features and human papillomavirus dna prevalence of warty and squamous cell carcinoma of the penis, Am J Surg Pathol 25 (2001), 673-678.
[75] L. Gregoire, A.L. Cubilla, V.E. Reuter, G.P. Haas and W.D. Lancaster, Preferential association of human papillomavirus with high-grade histologic variants of penile-invasive squamous cell carcinoma, J Natl Cancer Inst 87 (1995), 17051709.

[76] M. Frisch, C. Fenger and A.J. van den Brule, Variants of squamous cell carcinoma of the anal canal and perianal skin and their relation to human papillomaviruses, Cancer Res 59 (1999), 753-757.

[77] P.A. Poletti, A. Halfon and M.C. Marti, Papillomavirus and anal carcinoma, Int J Colorectal Dis 13 (1998), 108-111.

[78] K.R. Shroyer, C.G. Brookes, N.E. Markham and A.L. Shroyer, Detection of human papillomavirus in anorectal squamous cell carcinoma. Correlation with basaloid pattern of differentiation, Am J Clin Pathol 104 (1995), 299-305. 


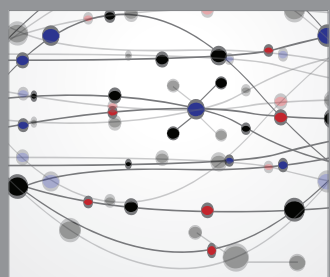

The Scientific World Journal
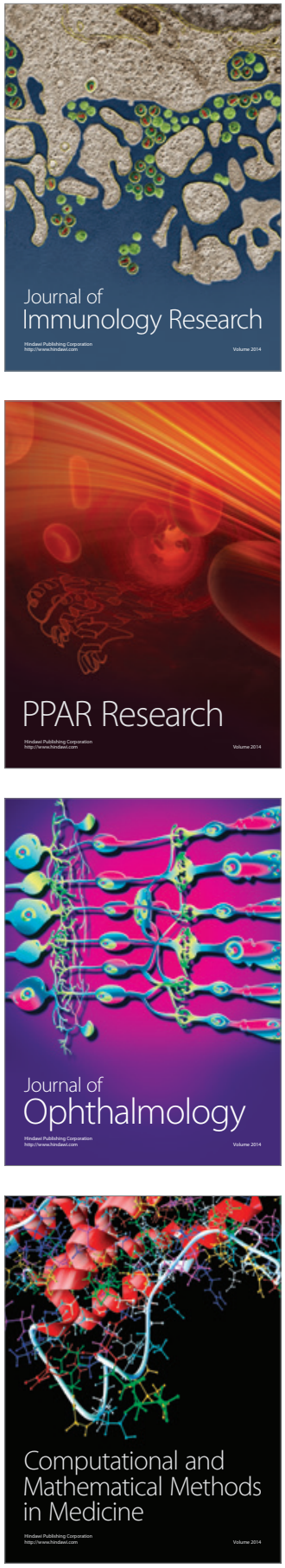

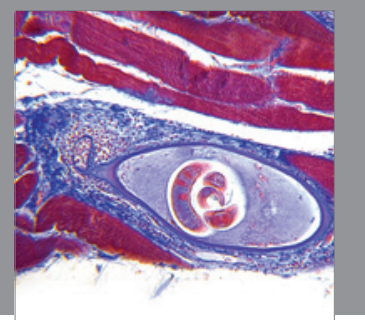

Gastroenterology

Research and Practice
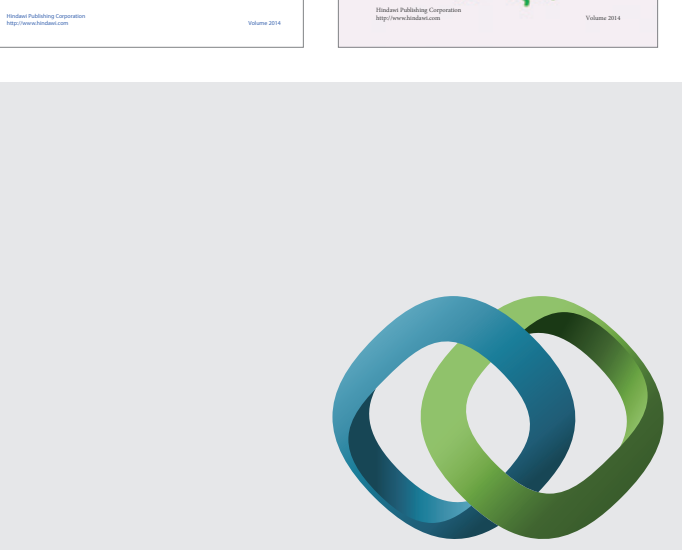

\section{Hindawi}

Submit your manuscripts at

http://www.hindawi.com
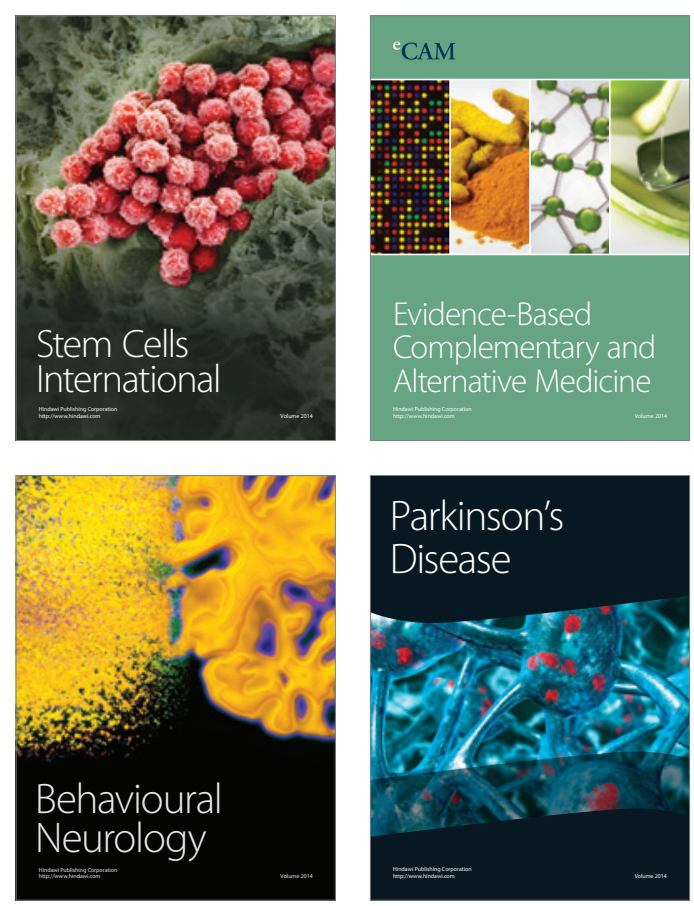

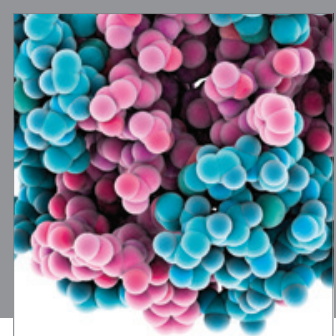

Journal of
Diabetes Research

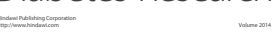

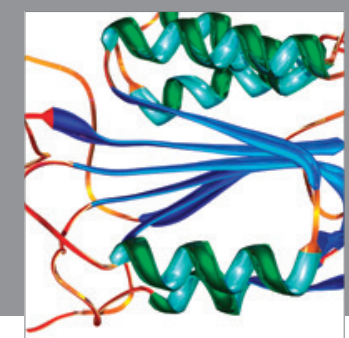

Disease Markers
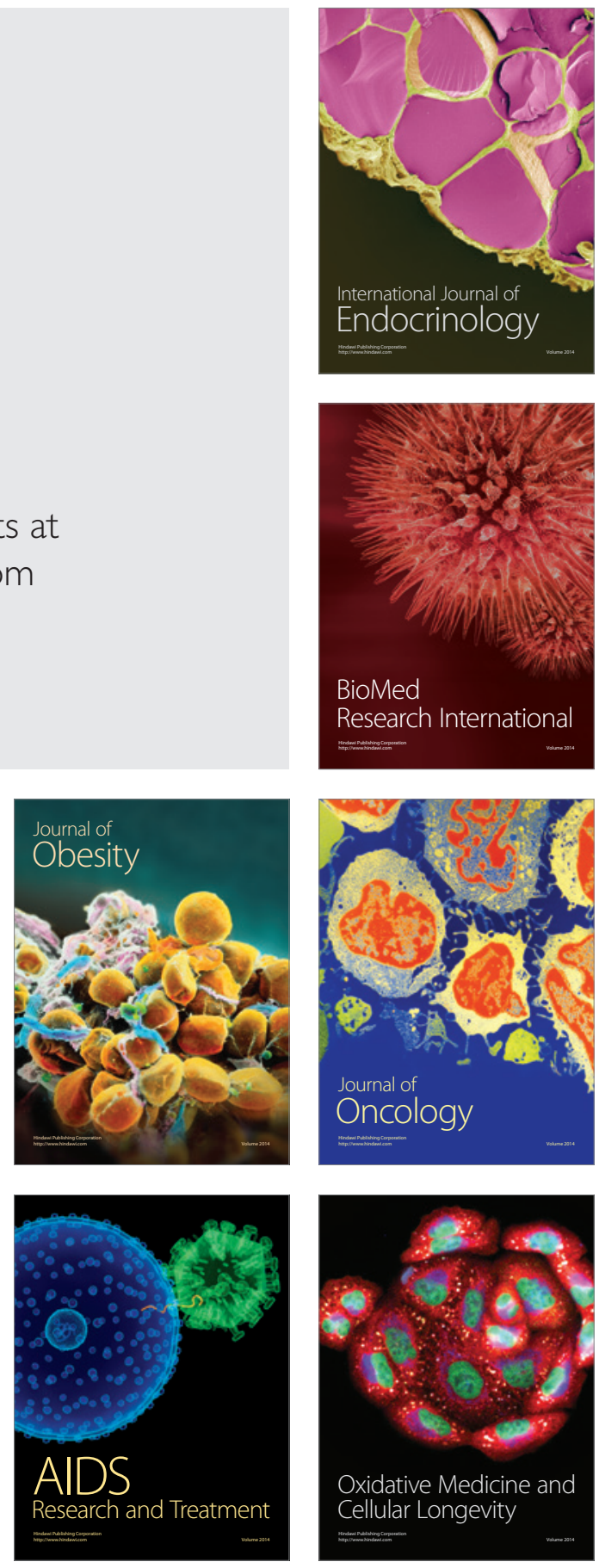